\title{
ON ANALYTIC CONTINUATION TO A SCHLICHT FUNCTION ${ }^{1}$
}

CARL H. FITZGERALD

This paper presents a characterization of the real functions on an interval of the real axis which can be analytically continued to be oneto-one functions of the upper half plane in to itself. The result depends heavily on work of Loewner.

In 1934, Loewner introduced the following notion [2]: a real, differentiable function $f$ on an interval $(a, b)$ of the real axis is monotonic of order $n \geqq 1$ means

$$
\sum_{i, j=1}^{n} \frac{f\left(x_{i}\right)-f\left(x_{j}\right)}{x_{i}-x_{j}} \xi_{i} \xi_{j} \geqq 0
$$

for all real $x_{i}$ in the interval $(a, b)$ and all real $\xi_{i}$, where

$$
\frac{f\left(x_{i}\right)-f\left(x_{i}\right)}{x_{i}-x_{i}}
$$

is identified with $f^{\prime}\left(x_{i}\right)$. The set of nonconstant functions which are monotonic of order $n$ is denoted by $\mathfrak{M}_{n}$; and the set of nonconstant functions which are monotonic of order $n$ for all $n \geqq 1, \bigcap_{n=1}^{\infty} \mathfrak{M}_{n}$, is denoted by $\mathfrak{M}_{\infty}$. The principal motivation for this concept is that $\mathfrak{M}_{\infty}$ is exactly the set of real functions on $(a, b)$ which can be analytically continued to be functions of the upper half plane into itself. Each of $\mathfrak{M}_{1}, \mathfrak{M}_{2}, \cdots, \mathfrak{M}_{\infty}$ is a pseudosemigroup with the operation being composition; consequently, the concept of infinitesimal transformations can be introduced. Loewner later considered the classes $\mathfrak{S}_{n}$ of functions which can be generated by the infinitesimal transformations of $\mathfrak{M}_{n}$, for $n=1,2, \cdots, \infty$. $\mathfrak{S}_{\infty}$ was shown to be the set of functions that can be analytically continued to be schlicht functions of the upper half plane into itself [3].

In a recent paper [4] Loewner proved that a necessary condition for a real function $f$ with positive derivative on a real interval $(a, b)$ to be in $\gamma_{n}, 1 \leqq n<\infty$, is that for all $x_{i}$ in $(a, b)$ and all real $\xi_{1}, \xi_{2}, \cdots, \xi_{n}$ such that $\sum_{i=1}^{n} \xi_{i}=0$,

$$
\sum_{i, j=1}^{n}\left[\log \frac{f\left(x_{i}\right)-f\left(x_{j}\right)}{x_{i}-x_{j}}\right] \xi_{i} \xi_{j} \geqq 0,
$$

Received by the editors May 24, 1966.

1 This work was partly supported by NSF Grant GP 5358 at Stanford University. 
where $\left(f\left(x_{i}\right)-f\left(x_{i}\right)\right) /\left(x_{i}-x_{i}\right)$ is again identified with $f^{\prime}\left(x_{i}\right)$. (Notice that since $f^{\prime}(x)>0$, the real branch of the logarithm may be chosen.) Loewner has conjectured that (2) is sufficient to insure that $f$ is in $\widetilde{S}_{n}$ for $2<n<\infty$.

In case $f$ is in $\mathfrak{S}_{\infty}$, it is clear that (2) must hold for all integers $n \geqq 1$, i.e. $\log ((f(s)-f(t)) /(s-t))$ is a positive semidefinite kernel on the space of continuous functions with compact support in $(a, b)$ and integral zero. The new result presented in this paper is that the converse is also true. In light of the connection cited between schlicht functions and $\mathfrak{S}_{\infty}$, these two facts can be stated as the following

THEOREM. Let $f$ be a real function with positive derivative in an interval $(a, b)$ of the real axis. $f$ can be analytically continued to be a schlicht. map of the upper half plane into itself if and only if

$$
\int_{a}^{b} \int_{a}^{b} \log \frac{f(s)-f(t)}{s-t} \phi(s) \phi(t) d s d t \geqq 0
$$

for all continuous functions $\phi$ having compact support in $(a, b)$ and such that $\int_{a}^{b} \phi(s) d s=0$.

It is only necessary to prove the sufficiency part of the theorem.

It is easily shown [5] that if on the space of $\left(\xi_{1}, \xi_{2}, \ldots, \xi_{n}\right)$ with $\xi_{i}$ real and $\sum_{i=1}^{n} \xi_{i}=0$, for all $x_{i}$ in some interval $(c, d)$ of the real axis,

$$
\sum_{i, j=1}^{n} H\left(x_{i}, x_{j}\right) \xi_{i} \xi_{j} \geqq 0,
$$

then on the space of all $\left(\xi_{1}, \xi_{2}, \cdots, \xi_{n}\right)$ with $\xi_{i}$ real, for $x_{i}$ in $(c, d)$,

$$
\sum_{i, j=1}^{n} \exp \left[H\left(x_{i}, x_{j}\right)\right] \xi_{i} \xi_{j} \geqq 0
$$

Hence (3) implies (1) is satisfied for all $n=1,2, \cdots$. (This statement is also an immediate consequence of representations (4) and (5) and the fact that the power series of the exponential function has only positive coefficients.) Therefore (2) $f$ can be analytically continued onto the upper half plane to be a function $F$ taking the upper half plane into itself. By the Schwarz reflection principle, it may be assumed that $F$ is also analytic in the lower half plane taking it into itself. To complete the proof it will be shown that $F$ is one-to-one.

For this purpose we begin by citing the following easily verified

LEMMA (BochNeR [1]). If $K(s, t)$ is a continuous function for $s$ and $t$ in $[\alpha, \beta]$, and 


$$
\int_{\alpha}^{\beta} \int_{\alpha}^{\beta} K(s, t) \phi(s) \phi(t) d s d t \geqq 0
$$

for all continuous functions $\phi$ such that $\int_{\alpha}^{\beta} \phi(s) d s=0$, then the function

$$
\begin{aligned}
L(s, t)= & K(s, t)-\mu \int_{\alpha}^{\beta} K(s, v) d v-\mu \int_{\alpha}^{\beta} K(u, t) d u \\
& +\mu^{2} \int_{\alpha}^{\beta} \int_{\alpha}^{\beta} K(u, v) d u d v
\end{aligned}
$$

is positive semidefinite on $[\alpha, \beta]$, where $\mu=1 /(\beta-\alpha)$.

Choose $\alpha$ and $\beta, a<\alpha<\beta<b$. Then (3) implies that the hypothesis of the lemma is satisfied if $K(s, t)$ is defined as the real branch of $\log ((f(s)-f(t)) /(s-t))$ for $s$ and $t$ in $[\alpha, \beta]$ and $K(s, s)$ is identified with $\log f^{\prime}(s)$. Since $L(s, t)$ is positive semidefinite and symmetric, Mercer's theorem implies that in the interval $[\alpha, \beta], L(s, t)$ is represented by the series

$$
\sum_{n=1}^{\infty} \frac{\psi_{n}(s) \psi_{n}(t)}{\lambda_{n}}
$$

where each $\psi_{n}(s)$ is an eigenfunction associated with a positive eigenvalue $\lambda_{n}$,

$$
\psi_{n}(s)=\lambda_{n} \int_{\alpha}^{\beta} L(s, t) \psi_{n}(t) d t
$$

and the series (5) converges uniformly for $s$ and $t$ in $[\alpha, \beta]$.

Let $D$ be the set of points off the real axis or in $[\alpha, \beta]$. Let $E$ be the set of points $(s, t)$ in $D \times D$ such that $s$ and $t$ are not in the same open half plane. $F(s)$ is analytic in $D$, and takes the upper half plane in to itself and the lower half plane into itself. Thus $K(s, t)$ $=\log ((F(s)-F(t)) /(s-t))$ can be defined by analytic continuation for all points $(s, t)$ in $E$. By equation $(4), L(s, t)$ can be continued to be analytic in $E$. Thus by equation $(6), \psi_{n}(s)$ can be continued to be analytic in $D$. It will be shown that for fixed $t$ in $D$ and $s$ in a compact subset of $D$, the series (5) converges uniformly; similarly, for $s$ fixed in $D$ and $t$ in a compact subset of $D,(5)$ converges uniformly.

Let $s_{0}$ be a point in the upper half plane, and $\Gamma$ be a differentiable Jordan curve in the upper half plane containing $s_{0}$ and having $\alpha$ and $\beta$ as end points. For each positive in teger $k$, consider a function $X_{k}(s)$ which is zero on $\Gamma$ except on an arc of length $1 / k$ containing $s_{0}$ where 
$X_{k}(s)=k$. By a theorem of Walsh [6] the polynomials are dense in the sense of the supremum norm topology in the space of continuous functions on $\Gamma$. By this theorem, there exist polynomials $P_{k}(s)$ such that

$$
\int_{\Gamma}\left|X_{k}(s)-P_{k}(s)\right| \cdot|d s| \leqq \frac{1}{k} .
$$

Let $\bar{\Gamma}$ be the reflection of the curve $\Gamma$ in the real axis, and $L_{m}(s, t)$ be the positive semidefinite kernel on $[\alpha, \beta]$ defined by

$$
L_{m}(s, t)=L(s, t)-\sum_{n=1}^{m} \frac{\psi_{n}(s) \psi_{n}(t)}{\lambda_{n}}
$$

for $(s, t)$ in $E$.

For $(s, t)$ in $E, L_{m}(s, t)$ is analytic in each variable when the other is fixed, hence the Cauchy integral theorem can be applied twice to give

$$
\int_{\bar{\Gamma}} \int_{\Gamma} L_{m}(s, t) P_{k}(s) \overline{P_{k}(\bar{t})} d s d t=\int_{\alpha}^{\beta} \int_{\alpha}^{\beta} L_{m}(s, t) P_{k}(s) \overline{P_{k}(t)} d s d t .
$$

Thus, since $L_{m}(s, t)$ is a positive semidefinite kernel on $[\alpha, \beta]$,

$$
\int_{\bar{\Gamma}} \int_{\Gamma} L_{m}(s, t) P_{k}(s) \overline{P_{k}(\bar{t})} d s d t \geqq 0 .
$$

Taking the limit as $k \rightarrow \infty$,

$$
L_{m}\left(s_{0}, \bar{s}_{0}\right) \geqq 0 .
$$

This inequality, (7) and the fact that $\psi_{n}(s)$ is real on $[\alpha, \beta]$ imply

$$
L(s, \bar{s}) \geqq \sum_{n=1}^{m} \frac{\left|\psi_{n}(s)\right|^{2}}{\lambda_{n}}
$$

for all $s$ in the upper half plane and all $m=1,2, \cdots$. By symmetry, (8) holds for $s$ in the lower half plane; and it has already been mentioned that by Mercer's theorem (8) holds for $s$ in $[\alpha, \beta]$.

For $s$ and $t$ in $D$, knowing $\lambda_{n}>0$, Schwarz's inequality gives

$$
\sum_{n=1}^{m}\left|\frac{\psi_{n}(s) \psi_{n}(t)}{\lambda_{n}}\right| \leqq\left[\sum_{n=1}^{m} \frac{\left|\psi_{n}(s)\right|^{2}}{\lambda_{n}}\right]^{1 / 2}\left[\sum_{n=1}^{m} \frac{\left|\psi_{n}(t)\right|^{2}}{\lambda_{n}}\right]^{1 / 2} .
$$

By (8), $\sum_{n=1}^{\infty}\left(\left(\left|\psi_{n}(s)\right|^{2}\right) / \lambda_{n}\right)$ converges to a finite quantity bounded by $L(s, \bar{s})$. Thus for $s$ fixed in $D$, given $\epsilon>0$, (9) implies for sufficiently large $l$, 


$$
\sum_{n=l}^{\infty}\left|\frac{\psi_{n}(s) \psi_{n}(t)}{\lambda_{n}}\right| \leqq \epsilon \cdot[L(t, \bar{t})]^{1 / 2}
$$

Since $L(t, \bar{t})$ is uniformly bounded on compact subsets of $D$, for $s$ fixed in $D$ and $t$ in a compact subset of $D$, the series (5) converges uniformly in $t$. And clearly if $t$ is fixed in $D$, and $s$ is in a compact subset of $D$, the series (5) converges uniformly in $s$.

Consequently, for $s$ and $t$ in $D$, the series (5) converges to a function which is analytic in $s$ for fixed $t$, and analytic in $t$ for fixed $s$. (A theorem of Hartogs implies that this function is holomorphic in $D \times D$, but it is not necessary to use this fact.) The set $E$ is the domain of definition of $L(s, t)$. Since $L(s, t)$ is analytic on the connected set $E$, and the series (5) converges to $L(s, t)$ for $s$ and $t$ in $[\alpha, \beta]$, it is possible to extend $L(s, t)$ to all of $D \times D$ by identifying $L(s, t)$ with the series (5). Thus for $s$ and $t$ in $D, L(s, t)$ is analytic in each variable when the other is fixed.

Since $K(s, t)$ has been shown to be analytic in $E$, the last three terms in equation (4) are analytic for $s$ and $t$ in $D$. Thus equation (4) and the preceding paragraph imply that $K(s, t)$ can be extended to $D \times D$ to be analytic in each variable when the other is fixed. If $t_{0}$ is a point of $D, K\left(s, t_{0}\right)$ can be continued to be analytic in $D$. In particular, $F(s)=F\left(t_{0}\right)$ implies $s=t_{0}$ since $\log \left(\left(F(s)-F\left(t_{0}\right)\right) /\left(s-t_{0}\right)\right)$ has no logarithmic singularities. Hence $F$ is one-to-one.

This work is to be part of a dissertation. I am indebted to Professor Loewner for his assistance in preparing this paper.

\section{REFERENCES}

1. S. Bochner, Hilbert distance and positive definite functions, Ann. of Math. 42 (1941), 647-656.

2. C. Loewner (K. Löwner), Über monotone Matrixfunktionen, Math. Z. 28 (1934), 177-216.

3. - Semigroups of conformal mappings, Seminar on Analytic Functions, Vol. 1, Institute for Advanced Study, Princeton, N. J., 1947.

4. - On schlicht-monotonic functions of higher order, J. Math. Anal. Appl. 14 (1966), 320-325.

5. I. J. Schoenberg, Metric spaces and positive definite functions, Trans. Amer. Math. Soc. 44 (1938), 530.

6. J. L. Walsh, Interpolation and approximation by rational functions in the complex domain, 4th rev. ed., Colloq. Publ., Vol. 20, Amer. Math. Soc., Providence, R. I., 1966.

StANFORD UNIVERSITY 